\title{
Scan Throughput
}

National Cancer Institute

\section{Source}

National Cancer Institute. Scan Throughput. NCI Thesaurus. Code C94974.

The number of scans performed in some unit of time. 